\title{
PROBIOTICS AND THEIR ATTRIBUTES IN HUMAN HEALTH THERAPY: REVIEW
}

\author{
Kabwanga Ismail Tijjani ${ }^{1} \boldsymbol{\mathbb { }}$, Munanura James ${ }^{2}$, Cansu Altın ${ }^{3}$ \\ 1,3 Graduate School of Natural and Applied Sciences, Department of Dairy Technology, Ankara \\ University-Turkey \\ 2 Department of Agriculture and Food Science, College of Agriculture, Animal Science and \\ Veterinary Medicine, University of Rwanda. Kigali-Rwanda
}

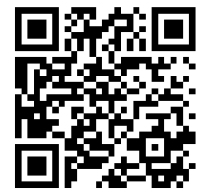

DOI: https://doi.org/10.29121/granthaalayah.v8.i5.2020.66

Article Type: Review Article

Article Citation: Kabwanga Ismail Tijjani, Munanura James, and Cansu Altın. (2020). PROBIOTICS AND THEIR ATTRIBUTES IN HUMAN HEALTH THERAPY: REVIEW. International Journal of Research GRANTHAALAYAH, 8(5), 158-164. https://doi.org/10.29121/granthaa layah.v8.i5.2020.66

Received Date: 10 May 2020

Accepted Date: 30 May 2020

Keywords:

Probiotic Microorganisms

Human Health Benefits

Mechanism

\section{ABSTRACT}

There is a strong correlation between diet and human health. Research shows that a number of disease and disorders can be prevented/regulated by the use of probiotic microorganisms. Probiotics are used in the management of illnesses/disorders such as intestinal pathogenic infections, helicobacter pylori infections, urogenital infection, cancer, obesity, Type 2 diabetes, GIT, IBD and lactose intolerance among others. Probiotics may also have a direct or indirect influence to the human health by the elimination of toxins, protection against enteric microorganisms and boosting the body immune system. These benefits among others have led to the in-depth studies aiming at the understanding of specific probiotics and the mechanism involved in disease therapy. Some commonly used probiotic strains in the dairy industry include among others Lactobacillus acidophilus, Bifidobacterium longum, Lactobacillus casei, Lactobacillus reuteri, and S. boulardii. Although the mechanisms used by probiotics in disease treatment is not specifically clear, its reported that the modification of the intestinal microbiota, production of short-chain fatty acids (SCFAs) hence maintenance of the $\mathrm{pH}$ in digestive system, production of bacteriocin and vitamins could be among the possible mechanisms. The aim of this review paper is to highlight the benefits of probiotics in human health \& infections control through the consumption of probiotic products and some mechanism involved.

\section{INTRODUCTION}

Probiotics are defined as viable mono or mixed culture of bacteria which, when ingested by human being or animal in adequate quantity provide health benefits to the host by improving the properties of the indigenous flora [1], [2], [3] Probiotics have active live cultures such as Lactobacillus acidophilus, Bifidobacterium longum, S. boulardii lactobacilli, Lactobacillus casei and Lactobacillus reuteri, among other bacteria that have been isolated from natural environments [4], [5] and some yeast strains like genus Saccharomyces and Bacillus clausii may also be used in probiotic products [4], [6].

The long safety history of lactic acid bacteria and bifidobacteria makes probiotic microorganisms to be considered as GRAS (generally regarded as safe) and QPS (Qualified Presumption of Safety) status in the European

(C) 2020 The Author(s). This is an open access article distributed under the terms of the Creative Commons Attribution License, which permits unrestricted use, distribution, and reproduction in any medium, provided the original author and source are credited. 
Union and United States as well [4], [7]. Probiotics exist in various foods but not limited to yoghurt, some cheese types, beverages, fermented vegetables and salty fishes [1], [8].

The health benefits of probiotics may include; the management of gastrointestinal disorders (GIT), obesity, Gastroenteritis, the production of antimicrobial compounds and regulation of cholesterol. A number of studies show a paramount impact of probiotics in the elimination of helicobacter, type-2 diabetes, anti- pathogenic, treatment of inflammatory bowel disease (IBD) and some allergic diseases [4],[5]

Table 1: Some Probiotic microorganisms used in human nutrition [4].

\begin{tabular}{|c|c|c|c|}
\hline Type Lactobacillus & Type Bifidobacterium & Other Lactic Acid Bacteria & Other Microorganisms \\
\hline L. acidophilus (a), ${ }^{*}$ & B. adolescentis (a) & Enterococcus faecium (a) & Bacillus clausii (a), \\
\hline L. amylovorus (b), ${ }^{*}$ & B. animalis (a), ${ }^{*}$ & Streptococcus & Escherichia coli Nissle 1917 (a) \\
\hline L. casei $(a),(b),{ }^{*}$ & B. bifidum (a) & thermophiles (a), ${ }^{*}$ & Saccharomyces cerevisiae \\
\hline L. gasseri $(\mathrm{a}),{ }^{*}$ & B. breve (b) & Lactococcus lactis(b)* & (boulardi) (a), ${ }^{*}$ \\
\hline L. helveticus (a), & B. infantis (a) & & \\
\hline L. johnsonii (b), ${ }^{*}$ & B. longum $(a), *$ & & \\
\hline L. pentosus (b),* & & & \\
\hline L. plantarum (b),* & & & \\
\hline L. reuteri (a), ${ }^{*}$ & & & \\
\hline L. rhamnosus (a),(b), & & & \\
\hline \multicolumn{4}{|c|}{ (a) Mostly as pharmaceutical products; } \\
\hline & & & \\
\hline
\end{tabular}

\section{BENEFITS OF PROBIOTICS IN HUMAN HEALTH}

\subsection{GASTROINTESTINAL INFECTIONS (GIT) CONTROL}

Probiotic microorganisms such as Lactobacillus and Bifidobacterium [6] have a great role in the prevention of gastrointestinal infections. This is affected through the production of lactic acid, adherence to the host cells, to exclude or reduce pathogenic bacteria and hydrogen peroxide. Production of bacteriocins which help to prevent the growth of pathogenic microbes and boosting the body's natural defense immunity [2], [5].

Bifidobacterium adolescentis, and Bifidobacterium pseudocatenulatum produce vitamin B groups for example; B1, B2, B3, B6, B8, B9, B12, which boost the immunity system, stimulate the generation of organic acids and amino acids. Probiotic produce enzymes like esterase, lipase, and co-enzymes A, Q, NAD, and NADP. Some products of probiotics' metabolism may also show antibiotic effect [2], [9]. Probiotic microbes also boost the immune system by the production of immunoglobulins, increase macrophages activity, the stimulation of $\gamma$-interferon production, influence the congenital and acquired immunological system through metabolites, components of the cellular wall and DNA recognized by the specialized host cells [10].

However, its advisable that the target probiotic bacteria should be subjected to in vitro tests like acid and bile tolerance, survival through the gastrointestinal tract, ability to adhere to gut epithelial tissue, temporary colonization, antagonism against pathogens and good technological properties [6].

\subsection{DIARRHEAL CONTROL}

Probiotics have a great role in diarrhea prevention for both the young and adults. Probiotic bacteria such as Lactobacillus GG, Bifidobacteria spp, Lactobacillus reuteri, and S. boulardii, have been used in the control of diarrhea. The above bacteria have the ability to suppress travelers' diarrhea by producing bacteriocins (nisin) and prohibiting diarrhea carrier microbes from binding to the epithelial cells [1], [8], [11]. Probiotics can prevent diarrhea through their ability to protect the host from toxins. Administering Saccharomyces boulardii yeast to acute and watery diarrhea patients led to their cure and reduced frequency complaint for two subsequent months [4]. 
Studies suggest that probiotic supplements may be used in the treatment of rotavirus-related diarrhea. Some authors reported the shortening in hospital stay period in patients given probiotics, but its important to know that most of these studies focus on possible beneficial short-term effects and not on long-term safety [1], [7].

\subsection{HELICOBACTER PYLORI INFECTIONS CONTROL}

Lactobacillus salivarius produce high amounts of lactic acid that can inhibit the growth of H. pylori in vitro. Probiotic bacteria are reported to have the ability to inhibit gastric colonization and activity of H. pylori related with peptic ulcers, gastric cancer and gastritis. L. salivarius inhibited H. pylori colonization in the in vitro studies and in mice as well. Furthermore, H. pylori infection may be regulated by probiotics though improving the eradication rate, tolerability and for the response of multiple antibiotics used in the treatment of helicobacter pylori infections [8]. The fact is that $\mathrm{H}$. pylori is

not only eradicated by probiotic treatment, but the number of bacteria may be reduced. In this case however, a combination of probiotics with antibiotics could increase the eradication rate and reduce the negative effects of the treatment [7].

\subsection{BLOOD CHOLESTEROL CONTROL}

Although cholesterol has an important role in the body like the synthesis of steroidal hormones, excessive blood cholesterol increases the risk of heart disease among others. The risk of heart attack is three times higher in those with hypercholesterolemia, compared to those who have normal blood lipid profiles [12]. Studies show that consumption of dairy products containing probiotics can reduce blood cholesterol [13]. In a 12 weeks randomized study where L. acidophilus CHO-220 and inulin was given to 32 hypercholesterolemic men and women, their plasma total cholesterol and low-density lipoprotein (LDL)-cholesterol lowered by 7.84 and 9.27\%, respectively [11]. The possible cholesterol reduction mechanism include; the production of SCFA's, the binding effect of cholesterol to probiotic cellular surface, deconjugation of bile via bile salt hydrolase, and last but not the least the incorporation of cholesterol molecules into the probiotic cellular membrane. Bifidobacterium and Lactobacillus species produce bile salt hydrolase (BSH) important in the hydrolysis of amide bonds between bile acids and their conjugates [14]. Studies show that BSH hydrolyze conjugated bile acids to form free primary bile acids from cholesterol which are less efficiently reabsorbed from the intestinal lumen and passed out in the fecal matter hence reduction of cholesterol [15]. It's important to note that Sterolibacterium denitrificans produces cholesterol dehydrogenase enzyme that helps to convert cholesterol to cholest-4-en-3-one and later into coprostanol which is excreted in the feces [16].

\subsection{LACTOSE INTOLERANCE CONTROL}

Lactose is the carbohydrate got from mammals' milk. Lactose intolerance or malabsorption may arise from insufficient or lack of lactase to convert lactose to glucose and galactose in the small intestine [17] leading to diarrhea, bloating and abdominal pain. Probiotic bacteria such as L. acidophilus milk and microbial ?-galactosidase in yogurt is important in lactose digestion and absorption in lactose intolerance patients [11], [12]

\subsection{PATHOGENIC ACTIVITY CONTROL}

Consumption of food contaminated with pathogens distorts the intestinal microbiota, causing food poisoning and other disorders in the body. Therefore the use of probiotics may help to prevent such pathogenic growth and regulate its side effects like toxins [4].

Probiotics have the ability to inhibit the growth of pathogens for example; Salmonella enterica, Serovar typhimurium, Clostridium perfringens, staphylococcus, E.coli and some species of Shigella [18], [19], [20]. The production of lactic acids, propionic, acetic, and butyric acid from the short-chain fatty acids (SCFAs), acetaldehydes, hydrogen peroxide (H2O2) by probiotics. The above compounds inhibit pathogenic microbe growth. These acids furthermore reduce the colonic lumen $\mathrm{pH}$ which is significant in the expression of numerous bacteria, enzymes and in the metabolism of carcinogens in the gut [6]. Probiotics also produce bacteriocins, ethanol, diacetyl, and peptides, 
all these help in the inhibition of pathogens. Peptides and bacteriocins are known to increase membrane permeability of the target cells leading to the depolarization of the membrane potential and hence cell death [21]. Furthermore, H2O2 causes the oxidation of sulfhydryl groups leading to the denaturation of several enzymes thus peroxidation of membrane lipid, increasing membrane permeability of the pathogenic microorganism and hence cell death [3]. Not only producing anti-pathogenic bioactive compounds that directly affect pathogens, probiotics also stimulate host anti- pathogenic defense pathways, such as the activation of the pathway involved in the production of defensins that are cationic anti-microbial peptides produced in several cell types including Paneth cells in the small intestine and intestinal epithelial cells. Probiotics confers exertion of anti-pathogenic activities by competing for pathogen binding or receptor sites and the available nutrients for pathogen growth [6].

\subsection{UROGENITAL INFECTION CONTROL}

According to the Centers for Disease Control and Prevention (CDCP), more than one billion women globally suffer from non-sexually transmitted urogenital infections such as; Urinary tract infection (UTI), bacterial vaginosis (BV), and several other yeast infections. Urogenital infection also include; Sexually transmitted diseases (STDs) such as gonorrhea, Candida and Chlamydia are a major cause of morbidity in the world [22]. These pathogenic microbes among others are becoming drug resistance demanding the use of non-pathogenic microbes to eliminate these pathogens [6].

Since is known that vaginal microbial flora affects the increased incidence of urinary tract infection (UTI). The lactic acid bacteria in the vagina such as; Lactobacillus delbrueckii subsp bulgaricus, Lactobacillus casei, Lactobacillus brevis, Lactobacillus vaginalis, Lactobacillus salivarius, Lactobacillus reuteri and Lactobacillus rhamnosus could have the ability to regulate the vaginal micro-environment. Vaginal microbial composition imbalance affects the health of the vaginal micro-environment to bacterial vaginosis (BV) and UTI. However, the use of lactic acid bacteria together with probiotics helps to balance the vaginal microbes hence reduction of infection risk [6], [7], [11], [23].

\subsection{DIABETIC MANAGEMENT}

Diabetes management is a multi-disciplinary medications with no single cure [24]. The $16 \mathrm{~S}$ rRNA gene sequencing, real-time PCR and fluorescent in situ hybridization studies show a correlation between the intestinal microbiota composition and metabolic disorder syndromes, like diabetes and obesity. The use of probiotics has an important role in the control of the disorder [25]. Gram-negative bacteroidetes and the Gram-positive firmicutes bacterial phyla dominat gut microenvironment. Studies show that obesity is associated with increased bacteroidetes over time, concurrent with a reduction in firmicutes. Type- 2 diabetes patients have a significant reduced number of firmicutes species in that the bacteroidetes/firmicutes ratio increases, which correlates positively with the plasma glucose concentration. Type- 2 diabetes can be managed by regulating gut hormones (gastric inhibitory polypeptide and glucagon- like peptide-1) with the use probiotic and prebiotic [6].

\subsection{OBESITY CONTROL}

Weight loss is majorly as a result of thermogenic and lipolytic responses stimulated in the sympathetic nervous system [26]. Studies show that probiotics such as Lactobacillus gasseri BNR17 reduces the increase of the adipocyte tissue which are the main source of leptin and adiponectin thus limiting leptin secretion. Other probiotic microbes such as Lactobacillus acidophilus, Bifidobacterium longum Lactobacillus casei have also been reported to have hypocholesterolemic effect [6], [26].

\subsection{PREVENTION OF INFLAMMATORY BOWEL DISEASE -IBD}

IBD comprises of the following disorders; Crohn's disease (CD), ulcerative colitis (UC) and the narrowing of the gastrointestinal tract hence abdominal pain, diarrhea and gastrointestinal bleeding [3], [24]. The etiology of those diseases is not clear. It's evident that they are mostly related to the chronic recurrent infections and or inflammations of the intestine [27]. 
The gut microbial imbalance has a great pathophysiological role in the positive regulation of IBD. However, this disorder can be altered by the use of probiotic supplements [28]. IBD is also related with the impaired production of SCFAs; butyrate, propionate and acetate to be specific. These SCFAs have an important role in the control of colonic homeostasis and improvement of the propulsive colonic function [6]

Some studies show that consumption of fermented dairy products prepared from Bifidobacterium lactis DN173010 in combination with Streptococcus thermophilus and Lactobacillus bulgaricus can activate gastrointestinal transit and increase distention and IBS signs and symptoms severity after 4 to 6 weeks of treatment [1].

Recent studies are concerned with the production of probiotic bacterial strains with the ability to produce and discharge immunomodulators like interleukin-10, trefoil factors (compact proteins co-expressed with mucins in the GIT, or lipoteichoic acid (a major constituent of the cell wall of Gram-positive bacteria) which can promote the host immune system thus restoring the level of protective commensal bacterial species [1], [6].

\subsection{CANCER CARE BY PROBIOTICS}

There is vast research on cancer regarding its pathology, genomics, proteomics and its public awareness. Probiotics have been reported to have anti-carcinogenic benefits [29]. Lactobacillus fermentum NCIMB-5221 and 8829 have been applied in vitro studies. It helped in suppression of colorectal cancer cells and promoted normal epithelial colon cell growth by the production of ferulic acid. L. acidophilus ATCC 314 and L. rhamnosus ATCC 51303 both were characterized with tumorigenic activity [6]. Moreover, probiotic strains like L. acidophilus LA102 and L. casei LC232 have also showed significant cytotoxic activities and within vitro anti-proliferative activity against two colorectal cancer cell lines (Caco-2 and HRT-18). Though probiotics could play a significant role in cancer control, there is a need for more detailed studies to ascertain its mechanism [30].

\subsection{EFFECT OF PROBIOTICS ON BRAIN AND CENTRAL NERVOUS SYSTEM -CNS}

The microbiota in the GIT affects both the GIT and gastrointestinal diseases. Several studies show the influence of gut microbiota on the CNS. The "microbiota-gut-brain axis" is an interactive bi-directional communication that ensures the exchange of regulatory signals between the GIT and CNS [31]. Clinical studies show an effect of probiotics on the CNS, because the gut microbiota influences the human brain development [32]. A daily intake of L. plantarum WCFS1 (4.5 _ $1010 \mathrm{CFU} /$ day) in children with autism spectrum disorder led to an improvement in their school records and attitude towards food [33], [34]. Another study showed a reduction in psychological distress in a randomized trial in healthy volunteers treated with oral administration of Lactobacillus helveticus R0052 and B. longum R0175. In addition, the use of L. casei strain Shirota in chronic fatigue syndrome patients showed a decrease in anxiety symptoms [6] due to the improvement of bowel function [35]. Controlled doses of probiotics such as; L. acidophilus W37, Bifidobacterium bifidum W2, L. salivarius W2, and L. casei W5 among others to healthy individuals showed an overall reduction in the cognitive reactivity to unhappy mood [36]. Though there is unclear information to justify the treatment of anxiety by probiotics, oral intake of L. acidophilus helps people to regulate their mood with concern to rewards and addictive behavior [6].

\section{CONCLUSION}

Probiotic microorganisms play essential role in human health. These microbes have been reported to have great therapeutic benefits in the treatment of several diseases and disorders such as; diarrhea associated with rotavirus, IBS, obesity, cholesterol, type 2 diabetes and food allergies. Also, probiotic may help in the treatment and prevention of cancer, enteritis, GIT disorders, bacterial infection and have a significant role in the management of lactose intolerance and diseases related to pathogens. There is a need for further studies to specify the exact probiotic strains, applicability in biomedical/clinical research and to explain the mechanisms by which probiotics confer beneficial effects on human health.

\section{SOURCES OF FUNDING}

None. 
Kabwanga Ismail Tijjani, Munanura James, and Cansu Altın

\section{CONFLICT OF INTEREST}

None.

\section{ACKNOWLEDGMENT}

None.

\section{REFERENCES}

[1] Rajyalakshmi, K., et al., Benefaction of probiotics in human gastro intestinal tract. 2019.

[2] Maldonado, N.C., et al., Identification, characterization and selection of autochthonous lactic acid bacteria as probiotic for feedlot cattle. Livestock science, 2018. 212: p. 99-110.

[3] Figueroa-González, I., et al., Probiotics and prebiotics-perspectives and challenges. Journal of the Science of Food and Agriculture, 2011. 91(8): p. 1341-1348.

[4] Markowiak, P. and K. Śliżewska, Effects of probiotics, prebiotics, and synbiotics on human health. Nutrients, 2017. 9(9): p. 1021.

[5] Shehata, M., et al., Screening of isolated potential probiotic lactic acid bacteria for cholesterol lowering property and bile salt hydrolase activity. Annals of Agricultural Sciences, 2016. 61(1): p. 65-75.

[6] Kerry, R.G., et al., Benefaction of probiotics for human health: A review. Journal of food and drug analysis, 2018. 26(3): p. 927-939.

[7] Halvorsen, R., et al., The use of probiotics for patients in hospitals. A benefit and risk assessment. Opinion of the Steering Committee of the Norwegian Scientific Committee for Food Safety. VKM Report, 2009.

[8] Amara, A. and A. Shibl, Role of Probiotics in health improvement, infection control and disease treatment and management. Saudi pharmaceutical journal, 2015. 23(2): p. 107-114.

[9] Nova, E., et al., Immunomodulatory effects of probiotics in different stages of life. British Journal of Nutrition, 2007. 98(S1): p. S90-S95.

[10] Oelschlaeger, T.A., Mechanisms of probiotic actions-a review. International Journal of Medical Microbiology, 2010. 300(1): p. 57-62.

[11] Daliri, E.B.-M. and B.H. Lee, New perspectives on probiotics in health and disease. Food Science and Human Wellness, 2015. 4(2): p. 56-65.

[12] Goh, Y.J. and T.R. Klaenhammer, A functional glycogen biosynthesis pathway in L actobacillus acidophilus: expression and analysis of the glg operon. Molecular microbiology, 2013. 89(6): p. 1187-1200.

[13] Ishimwe, N., et al., The perspective on cholesterol-lowering mechanisms of probiotics. Molecular nutrition \& food research, 2015. 59(1): p. 94-105.

[14] Lee, J.Y., et al., Proteomic and transcriptional analysis of Lactobacillus johnsonii PF01 during bile salt exposure by iTRAQ shotgun proteomics and quantitative RT-PCR. Journal of proteome research, 2013. 12(1): p. 432443.

[15] Huang, Y., et al., Lactobacillus plantarum strains as potential probiotic cultures with cholesterol-lowering activity. Journal of Dairy Science, 2013. 96(5): p. 2746-2753.

[16] Ooi, L.-G. and M.-T. Liong, Cholesterol-lowering effects of probiotics and prebiotics: a review of in vivo and in vitro findings. International journal of molecular sciences, 2010. 11(6): p. 2499-2522.

[17] Barling, P.M., Lactose tolerance and intolerance in Malaysians. IeJSME, 2012. 6(Suppl 1): p. S12-S23.

[18] Tejero-Sariñena, S., et al., Antipathogenic activity of probiotics against Salmonella Typhimurium and Clostridium difficile in anaerobic batch culture systems: is it due to synergies in probiotic mixtures or the specificity of single strains? Anaerobe, 2013. 24: p. 60-65.

[19] Saint-Cyr, M.J., et al., Use of the potential probiotic strain Lactobacillus salivarius SMXD51 to control Campylobacter jejuni in broilers. International journal of food microbiology, 2017. 247: p. 9-17.

[20] Chingwaru, W. and J. Vidmar, Potential of Zimbabwean commercial probiotic products and strains of Lactobacillus plantarum as prophylaxis and therapy against diarrhoea caused by Escherichia coli in children. Asian Pacific journal of tropical medicine, 2017. 10(1): p. 57-63.

[21] Islam, S.U., Clinical uses of probiotics. Medicine, 2016. 95(5). 
[22] Chan, P.A., et al., Extragenital infections caused by Chlamydia trachomatis and Neisseria gonorrhoeae: a review of the literature. Infectious diseases in obstetrics and gynecology, 2016. 2016.

[23] Waigankar, S.S. and V. Patel, Role of probiotics in urogenital healthcare. Journal of mid-life health, 2011. 2(1): p. 5.

[24] Iqbal, M.Z., et al., Probiotics and their beneficial effects against various diseases. Pakistan journal of pharmaceutical sciences, 2014. 27(2).

[25] Larsen, N., et al., Gut microbiota in human adults with type 2 diabetes differs from non-diabetic adults. PloS one, 2010.5(2).

[26] Karimi, G., et al., The anti-obesity effects of Lactobacillus casei strain Shirota versus Orlistat on high fat dietinduced obese rats. Food \& nutrition research, 2015. 59(1): p. 29273.

[27] Bengmark, S., Bioecological control of inflammatory bowel disease. Clinical Nutrition, 2007. 26(2): p. $169-181$.

[28] Kobyliak, N., et al., Probiotics in prevention and treatment of obesity: a critical view. Nutrition \& metabolism, 2016. 13(1): p. 14.

[29] Gayathri, D. and B. Rashmi, Anti-cancer properties of probiotics: a natural strategy for cancer prevention. EC Nutrition, 2016. 5(4): p. 1191-1202.

[30] Awaisheh, S., et al., In vitro cytotoxic activity of probiotic bacterial cell extracts against Caco-2 and HRT-18 colorectal cancer cells. Milk Science International-Milchwissenschaft, 2016. 69(7): p. 33-37.

[31] Mayer, E.A., K. Tillisch, and A. Gupta, Gut/brain axis and the microbiota. The Journal of clinical investigation, 2015. 125(3): p. 926-938.

[32] Tillisch, K., The effects of gut microbiota on CNS function in humans. Gut microbes, 2014. 5(3): p. 404-410.

[33] Takeda, S., et al., Antiallergic activity of probiotics from Mongolian dairy products on type I allergy in mice and mode of antiallergic action. Journal of Functional Foods, 2014. 9: p. 60-69.

[34] Palumbo, V.D., et al., The long-term effects of probiotics in the therapy of ulcerative colitis: A clinical study. Biomed Pap Med Fac Univ Palacky Olomouc Czech Repub, 2016. 160(3): p. 372-377.

[35] Szajewska, H., What are the indications for using probiotics in children? Archives of disease in childhood, 2016. 101(4): p. 398-403.

[36] Daliri, E., D. Oh, and B. Lee, Psychobiotics; a promise for neurodevelopmental therapy. J Probiotics Health, 2016. 4: p. $1 \mathrm{e} 4$. 